\title{
Intense Field Multiphoton Ionization via Complex Dressed States: Application to the H Atom
}

\author{
Shih-I Chu (a) \\ Joint Institute for Laboratory Astrophysics, University of Colorado and National Bureau of Standards, \\ Boulder, Colorado 80309 \\ and \\ William P. Reinhardt \\ Department of Chemistry, University of Colorado, Boulder, Colorado 80309, and Joint Institute \\ for Laboratory Astrophysics, University of Colorado and National Bureau of Standards, \\ Boulder, Colorado 80309 \\ (Received 30 August 1976; revised manuscript received 28 July 1977)
}

\begin{abstract}
Extension of Floquet theory to include continuum as well as bound atomic states yields a practical technique for computation of multiphoton ionization rates in the region where rms field strengths approach the strength of the internal atomic fields.
\end{abstract}

There is currently much interest in the problem of multiphoton ionization of atoms. ${ }^{1-3}$ At low fields perturbative techniques have been developed $^{1}$ and the theory is well in hand for the $\mathrm{H}$ atom." At higher fields the "generalized" cross sections become intensity dependent: Several groups have used higher-order perturbation theory, diagrammatic summation, and projection techniques to study these effects. ${ }^{3}$ However, within the framework of perturbation theory it is difficult to take into account the intensity-dependent broadening and shifting of the atomic levels in a straightforward and self-consistent manner as rms field strengths approach $10^{7}$ or $10^{8} \mathrm{~V} / \mathrm{cm}$ becoming a major perturbation on the atomic levels. We are thus led to seek a nonperturbative method for field strengths which are within a few orders of magnitude of the internal field strengths. That is, we seek a technique appropriate to the intermediate regime where fields are too high for conventional multiphoton perturbation theory, yet not high enough to reverse the procedure, treating the Coulomb interaction as a small perturbation on the motion of a charged particle in an intense ac field.

In the rf region of the spectrum the Floquet or "dressed-atom" model ${ }^{4}$ has been used in treatment of intense-field level shifts and anticrossings in the approximation that only finite numbers (often only two) of atomic states are directly involved. It is the purpose of this Letter to generalize the dressed-atom model to include the complete set of bound and continuum states of the bare atom. This has the effect of giving each of the dressed levels an intensity-dependent imaginary part (width) in addition to the usual field-induced shifts. Proper interpretation of the fre- quency and intensity dependence of these complex energies gives rise to rates for multiphoton processes without prior specification of the specific number of photons involved in the process, and is equivalent to infinite-order perturbation theory, self-consistent in that the shifts and widths of all levels are simultaneously determined.

Corresponding to the time-dependent Hamiltonian $H=H_{\text {(atomic) }}+\overrightarrow{\mathrm{F}} \cdot \overrightarrow{\mathrm{r}} \cos \omega t$ describing the interaction of an atom with a coherent monochromatic field, an equivalent time-independent Hamiltonian $\mathcal{H}_{\mathrm{F}}$ may be written in analogy with the semiclassical Floquet Hamiltonian of Shirley。 ${ }^{4}$ The resulting block structure is shown in Fig. 1, where the $V_{l, l}$,'s are dipole-coupling elements and the angular momentum blocks $\tilde{S}, \widetilde{P}, \tilde{D}, \ldots$ represent the projection of the atomic-electronic Hamiltonian onto states of total $L=0,1,2, \ldots$, etc. Thus, for example, in the case of the $\mathrm{H}$ atom the $\tilde{S}$ block consists of the $1 s, 2 s, 3 s, \ldots$, $n s, \ldots$ bound states and the entire $k s$ Coulomb continuum. The Hamiltonian of Fig. 1 has no discrete spectrum, and the existence of Floquet solutions of the time-dependent Schrödinger equation is not established. ${ }^{5}$ However, writing the time-evolution operator as

$$
\exp \left(-i \mathcal{H}_{\mathrm{F}} t / \hbar\right)=\frac{1}{2 \pi i} \int_{C} d z \frac{e^{-i z t / \hbar}}{z-\mathcal{H}_{\mathrm{F}}}
$$

gives the usual result $\mathrm{t}^{\mathrm{b}}$ that the time dependence is dominated by poles of $\left(z-\mathcal{H}_{F}\right)^{-1}$ near the real axis but on higher Riemann sheets, and that the complex energies of the poles are related to the positions and widths of the shifted and broadened dressed states. These complex-pole positions may be found directly from the analytically continued Floquet Hamiltonian, $\mathfrak{H C}_{\mathrm{F}}(\theta)$, obtained by 

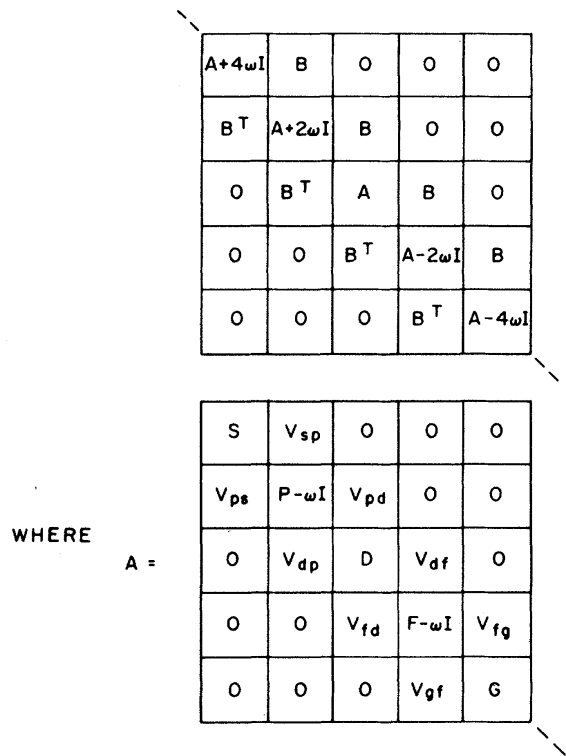

$A N D \quad B=$\begin{tabular}{|c|c|c|c|c|}
\hline 0 & 0 & 0 & 0 & 0 \\
\hline$v_{p s}$ & 0 & $v_{p d}$ & 0 & 0 \\
\hline 0 & 0 & 0 & 0 & 0 \\
\hline 0 & 0 & $v_{f d}$ & 0 & $v_{f g}$ \\
\hline 0 & 0 & 0 & 0 & 0 \\
\hline
\end{tabular}

FIG. 1. Structure of the Floquet Hamiltonian in the electric dipole approximation. The Hamiltonian is composed of Floquet blocks, of type A, which are in turn composed of angular momentum blocks $\widetilde{S}, \widetilde{P}, \widetilde{D}, \ldots$; both types of blocks are coupled by the (dipole) coupling elements $V_{l, l \pm 1}$.

the dilatation transformation ${ }^{6} r-e^{i \theta} r$. This transformation effects an analytic continuation of $\left(z-\mathfrak{H}_{\mathrm{F}}\right)^{-1}$ into the lower half-plane on appropriate higher Riemann sheets, allowing the complex dressed states to be determined by solution of a non-Hermitian eigenvalue problem. In practice the atomic blocks $\tilde{S}, \tilde{P}, \tilde{D}, \ldots$ were discretized ${ }^{7}$ by use of a finite subset of the complete discrete Laguerre basis $r^{l+1} e^{-\lambda r / 2} L_{n}{ }^{2 l+2}(\lambda r), n=0,1,2, \ldots$ which gives a Pollaczek ${ }^{7}$ quadrature representation of the bound and continuum contributions to the spectral resolution of the hydrogenic Hamiltonian.

As the techniques of dilatation transformation and $L^{2}$ discretization are unfamiliar within the context of intense-field effects, we illustrate the methods with a calculation of the dc Stark width and shift of the $1 \mathrm{~s}$ state of the $\mathrm{H}$ atom. In this case the dilatation transformation gives ${ }^{8}$

$$
\mathfrak{H C}_{\mathrm{F}} \rightarrow \mathcal{H}_{\mathrm{F}}(\theta)=H_{\text {atomic }}\left(r e^{i \theta}\right)+e^{i \theta} \overrightarrow{\mathrm{F}} \cdot \overrightarrow{\mathrm{r}},
$$

where

$$
\mathcal{H}_{\text {atomic }}\left(r e^{i \theta}\right)=e^{-2 i \theta} \frac{1}{2}\left(-\frac{d^{2}}{d r^{2}}+\frac{l(l+2)}{r^{2}}\right)-\frac{e^{-i \theta}}{r},
$$

and the Floquet matrix reduces to block $A$ of Fig. 1 , with $\omega=0$. Convergence to the complex eigenvalue adiabatically connected to the exact $1 s$ state at $F=0$ is illustrated in Table I, where convergence as a function of basis set (and number of angular momentum blocks) is shown.

Application to multiphoton ionization of hydrogen follows the same procedure as in the dc Stark case, except that now convergence must be obtained with respect to the number of Floquet blocks, ${ }^{9}$ as well as with respect to basis size and number of angular momentum blocks. To the extent that enough Floquet blocks are included to obtain convergence, all relevant processes involving differing photon numbers are simultaneously and self-consistently included. For example, Table II gives ionization cross sections in the frequency region where a single photon is sufficient for ionization. These flux-dependent cross sections were computed from the transition rate implied by the imaginary part of the dressed $1 \mathrm{~s}$

TABLE I. Convergence (Ref. 8) of the complex eigenvalue (in a.u.) representing the broadened and shifted " $1 s$ " state of the $\mathrm{H}$ atom as a function of the number of angular momentum blocks $\left(N_{l}\right)$ and number of basis functions $(n)$ for a dc field $F=0.1$ a.u. $\left(5.1 \times 10^{8} \mathrm{~V} / \mathrm{cm}\right)$. Hehenberger et al. give $-0.527-0.72 \times 10^{-2} i$ for this pole position. ${ }^{a}$

\begin{tabular}{rcc}
\hline \multicolumn{1}{c}{$N_{l}=4$} & $N_{l}=6$ \\
\hline 5 & $-0.52728-0.7154 \times 10^{-2} i$ & $-0.52731-0.7259 \times 10^{-2} i$ \\
8 & $-0.52739-0.7234 \times 10^{-2} i$ & $-0.52741-0.7273 \times 10^{-2} i$ \\
12 & $-0.52740-0.7234 \times 10^{-2} i$ & $-0.52742-0.7270 \times 10^{-2} i$ \\
\hline
\end{tabular}

${ }^{\mathrm{a}}$ M. Hehenberger et al., Phys. Rev. A $\underline{10}, 1494$ (1974). 
TABLE II. Intensity-dependent ionization cross section $\left(a_{0}{ }^{2}\right)$ for the dressed " $1 s$ " state as a function of frequency, $\omega$, and field strength, $F_{\mathrm{rms}}$ 政 atomic units (a.u.) $\hbar=m_{e}=e^{2}=1$, and $\omega=0.5$ is the zero-field ionization threshold. $M_{\mathrm{F}}$ is the number of Floquet blocks needed to achieve convergence. Nonlinear effects are seen to be quite small in this frequency region. The intense-field corrections at $F_{\mathrm{rms}}=0.05$ a.u. are of the order of magnitude predicted by Zernik and Klopfenstein (Ref. 10) and Karule (Ref. 10) using second-order perturbation theory, but detailed comparison with the results of these authors is difficult as (1) they neglect field-induced level shifts of the $1 s$ state, and (2) we have followed the time dependence of the adiabatically prepared "dressed" $1 s$ state, rather than the bare hydrogenic $1 s$ state as is implicit in the perturbation work.

\begin{tabular}{|c|c|c|c|}
\hline$\omega F_{\mathrm{rms}}{ }^{\mathrm{a}}$ & $\begin{array}{c}10^{-4} \\
\left(M_{\mathrm{F}}=3\right)\end{array}$ & $\begin{array}{c}0.05 \\
\left(M_{\mathrm{F}}=4\right)\end{array}$ & $\begin{array}{c}0.20 \\
\left(M_{\mathrm{F}}=5\right)\end{array}$ \\
\hline 0.5 & 0.2250 & 0.2282 & 0.2534 \\
\hline 0.6 & 0.1378 & 0.1384 & 0.1418 \\
\hline 2.0 & 0.0044 & 0.0044 & 0.0044 \\
\hline
\end{tabular}

${ }^{\mathrm{a}} F_{\mathrm{rms}}$ is given in $\mathrm{a}_{0} \mathrm{u}_{\circ}\left(1 \mathrm{a}_{\mathrm{o}} \mathrm{u}_{0}=5.1 \times 10^{9} \mathrm{~V} / \mathrm{cm}\right.$, which corresponds to an intensity of $7.0135 \times 10^{16} \mathrm{~W} / \mathrm{cm}^{2}$ ).

state, and are, at low fields, intensity independent and identical to the usual photoeffect cross section.

Figure 2 shows the intensity dependence of the generalized two-photon ionization cross section, ${ }^{2}$ $\hat{\sigma}_{2} \equiv(\text { ionization rate)/(intensity) })^{2}$, of the bare $1 s$ state in the neighborhood of the $1 s \rightarrow 2 p$ and $1 s \rightarrow 3 p$ one-photon resonances. The time evolution of the bare $1 s$ state was determined from $\exp \left(-i \mathcal{H}_{\mathrm{F}} t\right)$ $\hbar)|1 s\rangle$ via Eq. (1), corresponding to a sudden turning on of the field at $t=0$, the contour integral being calculated from the matrix spectral resolution of $\left[z-\mathfrak{H}_{\mathrm{F}}(\theta)\right]^{-1}$. At low fields $\left(F \lesssim 10^{-4}\right.$ a.u. $), \hat{\sigma}_{2}$ is independent of field strength, except very close to resonance, and to our working accuracy (convergence to better than $1 \%$ ) identical with perturbation results. As the fields approach 1 or $2 \%$ of atomic field strengths the intermediate $2 p$ state broadens substantially and the strong near-resonant enhancement near $\omega=0.375$ is quenched; however, off resonance $\hat{\sigma}_{2}$ remains essentially field independent. At the higher fields shown in Fig. 2 the definition of $\hat{\sigma}_{2}$ depends on the choice of time scales ${ }^{12}$; the results presented here follow from initial rates determined by averaging over many Rabi cycles. ${ }^{12}$ Although the number of Floquet blocks needed for convergence increases as a function of $F_{\mathrm{rms}}$, we have empirically

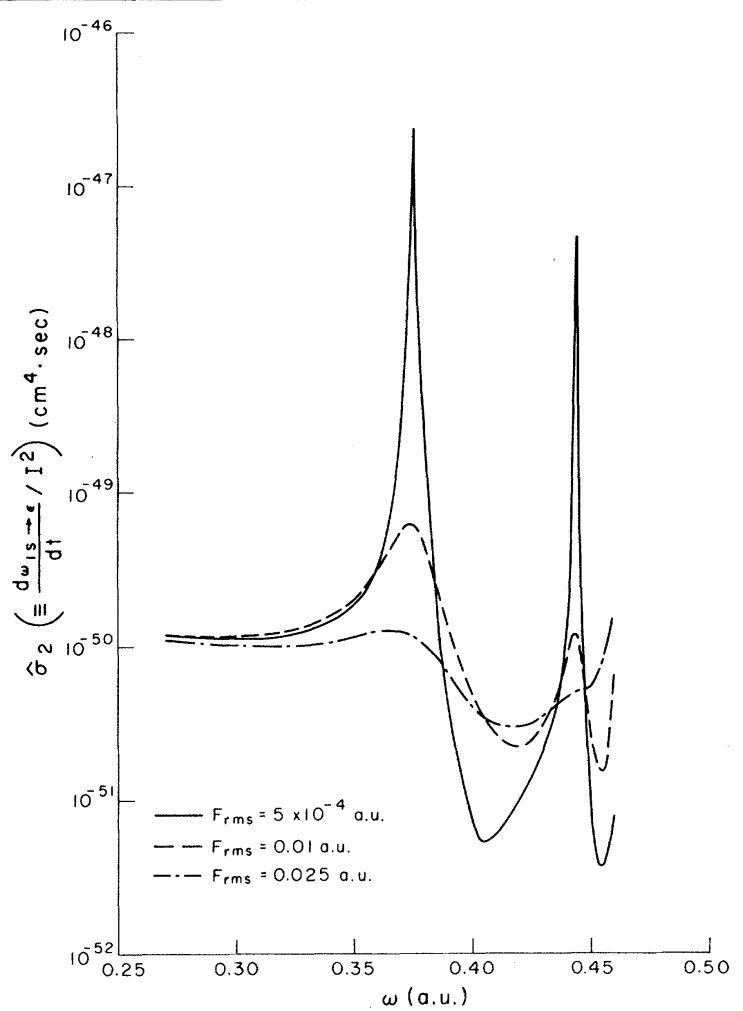

FIG. 2. Intensity-dependent generalized cross section, $\hat{\sigma}_{2}$, for ionization of the bare $1 s$ state of $\mathrm{H}$ in the frequency region dominated by resonant two-photon ionization. It is seen that at high fields the near-resonant enhancement is quenched, and that the nonresonant cross section is, to a very good approximation, field independent even at an intensity of $\sim 10^{13} \mathrm{~W} / \mathrm{cm}^{2}$. The intensity dependence of the resonant line shape is qualitatively in agreement with that of the two-state-pluscontinuum model of Ref. 11 .

found that resonant and off-resonant cross sections converge with four to five blocks for fields up to 10 to $20 \%$ of atomic field strengths (intensities up to $\sim 7 \times 10^{14} \mathrm{~W} / \mathrm{cm}^{2}$ ).

The authors acknowledge the support of the National Science Foundation and the U. S. Energy Research and Development Administration, stimulating conversations with Dr. P. L. Knight and Dr.A. Szöke, and the computational acumen and assistance of Chela V. Kunasz and Linda Kaufman.

\footnotetext{
(a) Present address: Department of Physics, Yale University, New Haven, Connecticut 06520.

${ }^{1}$ Recent reviews are J. S. Bakos, Adv. Electron. Electron Phys. 36, 57 (1974); P. Lambropoulos, Adv. At. Mol. Phys。 12 , 87 (1976); "Multiphoton Processes," edited by J. Eberly and P. Lambropoulos (Wiley, New
} 
York, to be published).

${ }^{2}$ For example, W. Zernik, Phys. Rev. 135, A51 (1964); H. B. Bebb and A. Gold, Phys。Rev. 143, 1 (1966); T. N. Chan and C. L. Tang, Phys. Rev. 185, 42 (1969); Y. Gontier and M. Trahin, Phys. Rev. A $\underline{5}$, 1896 (1971); E. Karule has reviewed work on $\mathrm{H}$ through 1976 in "Multiphoton Processes," edited by J. Eberly and P. Lambropoulos (Wiley, New York, to be published).

${ }^{3}$ Y. Gontier, N. K. Rahman, and M. Trahin, Phys. Rev. Lett。34, 779 (1975), and Phys。Rev. A 14, 2109 (1976); S. Yeh and P. Stehle, Phys. Rev. A 15,213 (1977); S. Swain, J. Phys. A 8,1277 (1975); F. H. M. Faisal, J. Phys. B 9 , 3009 (1976); L. Rosenberg, Phys. Rev. A 14, 1137 (1976).

${ }^{4}$ S. Autler and C. H. Townes, Phys. Rev. 100, 703 (1955); J. H. Shirley, Phys. Rev. B 138, 979 (1965); C. Cohen-Tannoudji and S. Haroche, J. Phys. (Paris) $\underline{30}, 125,153$ (1969).
${ }^{5}$ R. H. Young, W. J. Deal, and N. R. Kestner, Mol。 Phys。17, 369 (1966); Ya。Zel'dovich, Usp。Fiz。Nauk 110,139 (1973) [Sov。Phys。Usp。16, 427 (1973)]。

${ }^{6} \mathrm{~A}$ mathematical review designed for physicists has been given by B. Simon, Ann。 Math。Stat。97, 247 (1973)。

${ }^{7}$ H. A。 Yamani and W。 P. Reinhardt, Phys。 Rev。A 11, 1144 (1975)。

${ }^{8}$ W. P. Reinhardt, Int。 J。Quantum. Chem。 S10, 359 (1976).

${ }^{9}$ Truncation in terms of Floquet blocks is closely related to limiting the excursions in the walks of Yeh and Stehle (Ref。3)。

${ }^{10} \mathrm{~W}$. Zernik and R。W.Klopfenstein, J。 Math。 Phys。 (N.Y.) 6, $262(1965)$; E。 Karule, to be published, and in "Multiphoton Processes," edited by J. Eberly and P. Lambropoulos (Wiley, New York, to be published)。

${ }^{11} \mathrm{~S}_{\circ}^{\prime}$ Feneuille and L。Armstrong, J. Phys。(Paris) 36, L235 (1975)。

${ }^{12} \mathrm{~S}$ 。-I Chu and W。 P. Reinhardt, to be published.

\title{
Angle-Dependent Reflectance of Laser-Produced Plasmas
}

\author{
R. P. Godwin, R. Sachsenmaier, and R. Sigel \\ Projektgruppe für Laserforschung der Max-Planck-Gesellschaft zur Förderung der Wissenschaften, \\ D-8046 Garching, Federal Republic of Germany \\ (Received 10 August 1977)
}

\begin{abstract}
The reflectance of planar targets irradiated by $400-\mathrm{mJ}, 30-\mathrm{ps}$ Nd: glass laser pulses was measured as a function of angle of incidence and polarization with an Ulbricht spherical photometer. At normal incidence we find reflectances $>0.6$. The reflectance for $p$-polarized light has a minimum at an incidence angle of $20^{\circ}$ to $30^{\circ}$; for angles greater than $60^{\circ}$ total reflection occurs for both $s$ - and $p$-polarized light. The results seem consistent with simulations.
\end{abstract}

Understanding the absorption of intense laser radiation incident upon solid targets is of basic importance in laser-fusion studies. Optical energy-balance measurements are the most direct means for gaining information. Recent measurements with planar targets ${ }^{1-3}$ have shown reflectances of 0.5 and more at normal incidence. These observations have created renewed interest in reflectance measurements as a function of angle of incidence and polarization which should yield more information than do normal-incidence measurements. In particular, optical resonance ${ }^{4-6}$ should enhance absorption of $p$-polarized light.

Measurements at normal incidence have indicated polarization effects in the scattered laser light, but these are rather indirect and difficult to correlate with theoretical models. ${ }^{2}$ Ion-velocity measurements at a fixed angle of incidence give qualitative evidence for a polarization effect, but no quantitative measure of absorption. ${ }^{7}$ In this Letter we report direct reflectance measure- ments as a function of angle of incidence and polarization which exhibit the polarization effects expected on the basis of optical resonance. In a previous experiment ${ }^{8}$ these effects were not evident.

In laser-produced-plasma experiments a large fraction of the nonabsorbed laser light may be scattered in a rather diffuse manner. ${ }^{1}$ Hence a reflectometer to be used in such experiments should have a $4 \pi$ collection angle, especially if the target is tilted during the measurements. An Ulbricht spherical photometer ${ }^{9}$ is ideal. It consists of a hollow sphere with its inner surface coated by a diffuse reflector. The attractive feature of such a device is that the measured intensity is independent of the angular radiation distribution of the source (in our case, the reflecting laser-produced plasma).

Our Ulbricht sphere was constructed by painting the inside of a 150-mm-diam plastic shell with Eastman white reflectance paint (see Fig. 1). Ap- 